\section{A new variant of chromosome 3 with unusual staining properties}

Large heterochromatic regions in autosomes other than 1 , 9 , and 16 occur sporadically but have not previously been

Received for publication 21 January 1988. Accepted for publication 1 February 1988. reported for chromosome 3 . The centromeric region (p11-q11) of chromosome $3^{1}$ has been shown to stain darkly with GTG and CBG banding and variably with QFQ banding. It is variable in size and centromere position $^{2}$ within the heterochromatic band.

Analysis of lymphocyte chromosomes of a male whose wife had had three spontaneous miscarriages following the birth of a normal male child showed an anomalous chromosome 3 in an otherwise normal male karyotype. The variant chromosome 3 (figure) had a large GTL
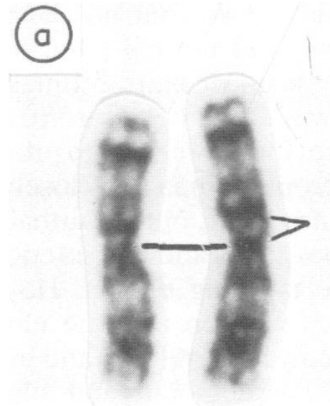

GTL
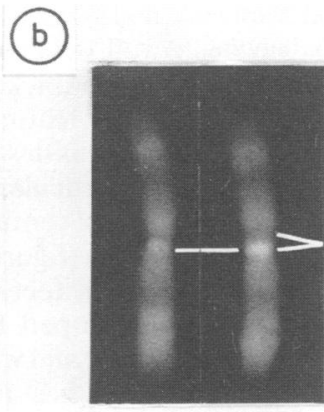

QFQ

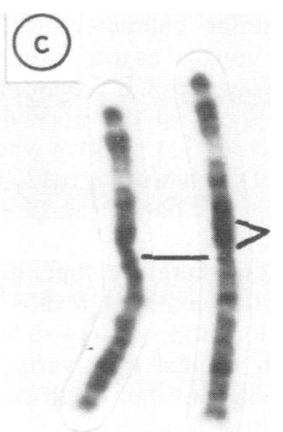

GBG
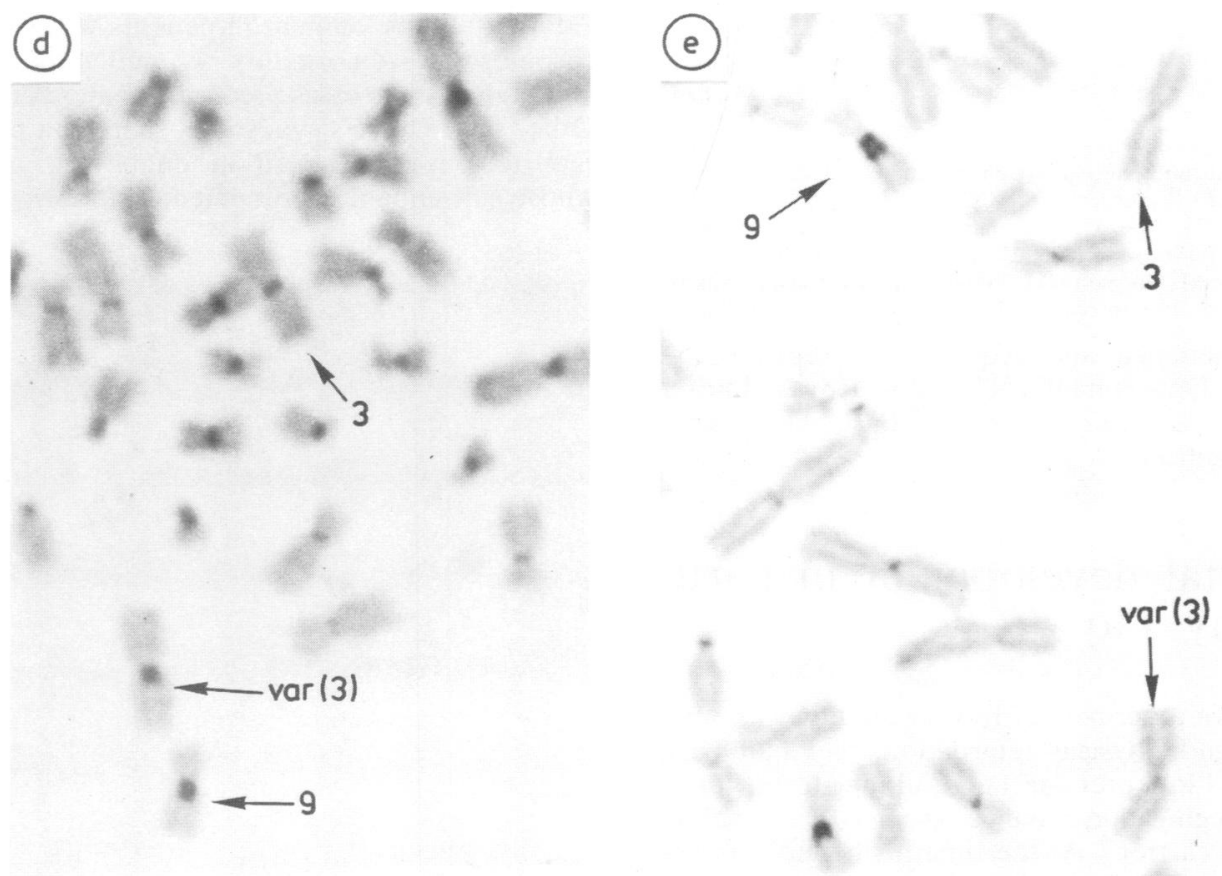

FIGURE Partial karyotype showing chromosomes 3 of the proband after (a) GTL, (b) QFQ, (c) GBG, (d) CBG, and (e) G11 banding. 
negative region in the short arm proximal to the centromere. The region stained positively with $\mathrm{CBG}$ and GBG banding indicating that it is heterochromatic and late replicating. It does not fluoresce brilliantly with QFQ banding in contrast to the centromeric region and it is G11 negative, indicating that it is not translocated 9 heterochromatic material. Investigation of parental chromosomes revealed that the variant was maternally inherited. The mother had had three normal children and no evident miscarriages. The karyotype of the proband might be described as 46,XY, $\operatorname{var}(3)(\mathrm{p} 11+)$ mat.

The staining characteristics of the presented variant region are unusual as it stains negatively with GTL banding but positively with $\mathrm{CBG}$ banding. The only $\mathrm{G}$ negative, $\mathrm{C}$ positive regions in the normal human karyotype are at 9 p12 (which is G11 positive whereas the variant described here is not) and within 1q12, where alternating positive and negative $G$ bands can be observed in prometaphase chromosomes. ${ }^{1}$

It is likely therefore that the observed GTL negative, CBG positive material results from amplification rather than translocation, as has also been suggested by Seabright et $a l^{\beta}$ with respect to a variant of chromosome 5 .

No conclusions can be drawn regarding an association between this variant and the presentation of the proband, namely recurrent abortion in his wife, particularly as the variant is also present in the mother who reproduced normally.

V Petrovic

Department of Genetics,

Royal Children's Hospital, Melbourne, Australia.

\section{References}

1 ISCN. An international system for human cytogenetic nomenclature. Birth Defects 1985;XXI(I).

${ }^{2}$ Phillips RB. New C band markers of human chromosomes: C band position variants. J Med Genet 1980;17:380-5.

${ }^{3}$ Seabright M, Gregson NM, Johnson M. A familial polymorphic variant of chromosome 5. J Med Genet 1980;17:444-6.

Correspondence and requests for reprints to $\mathrm{V}$ Petrovic, Department of Genetics, Royal Children's Hospital, Flemington Road, Parkville, Victoria 3052, Australia.

\section{Pubertal development in partial trisomy $14 \mathrm{q}$}

In 1980 we described an 18 year old boy with severe mental and physical retardation who had partial trisomy $14 q$ (pter $\rightarrow q 21$ ) resulting from tertiary trisomy segregation in meiosis from a $t(3 ; 14)(\mathrm{p} 25 ; \mathrm{q} 21)$ maternal carrier. ${ }^{1}$ At the time his height was 114.5 $\mathrm{cm}$ (on the 50th centile for a five year old), weight 22 $\mathrm{kg}$, and head circumference $49 \mathrm{~cm}$ (on the 50th

Received for publication 18 November 1988.

Revised version accepted for publication 17 February 1988. centile for a two year old). He had no pubertal:

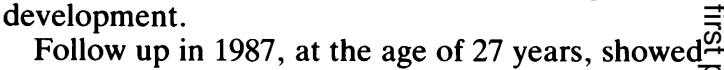
a length of $140 \mathrm{~cm}$ (on the 50th centile for a $101 / 2$ ? year old), bare weight $27.4 \mathrm{~kg}$, and head circum- $\frac{\bar{\sigma}}{\bar{c}}$ ference $50 \mathrm{~cm}$ (on the 50 th centile for a three and a $\frac{\rho}{\Phi}$ half year old). Hand length was $13 \mathrm{~cm}$ (with palm气 length $8.5 \mathrm{~cm}$ ), foot length $16 \mathrm{~cm}$, and chestis circumference $74 \mathrm{~cm}$. Pubertal development was $\vec{\circ}$ complete with coarse skin, rough facial hair, adult: male axillary and pubic hair (but no chest hair), $\mathrm{a} \vec{\omega}$ well developed penis, and normal adult testes. $\mathrm{He}$ ? had a thick, healthy head of hair and masculinised facial features. He was shaved every third day. The i deformities noted previously were more pronounced, $\stackrel{\sim}{-}$ in particular the microphthalmia and closely set eyes-(inner canthal distance $2 \mathrm{~cm}$, outer canthal distance $\infty$ $7 \mathrm{~cm}$ ) (figure), the pes cavus and talipes equinovarus of the feet, and the tapering fingers. He had also응 developed flexion contractures of the elbows and knees and was markedly hyperreflexic and hypertonic.o

Studies included haemoglobin and blood film, renal and liver function tests, serum electrolytes, and thyroid function tests which were all within足 the normal male adult range, as was the serum $\overrightarrow{ }$ testosterone $(21 \mathrm{nmol} / \mathrm{l}), \mathrm{FSH}(11.9 \mathrm{mIU} / \mathrm{ml})$, a LH $(6.0 \mathrm{mIU} / \mathrm{ml})$.

Pubertal development in patients with autosonalo chromosome abnormality is generally delayed, although in some cases precocious puberty occurs. ${ }^{2}$ 긍 Other than Down's syndrome, there is a paucity of reported cases detected in infancy or childhood $\stackrel{\AA}{\mathscr{Q}}$ whose subsequent development is followed. In one $\overrightarrow{\vec{O}}$

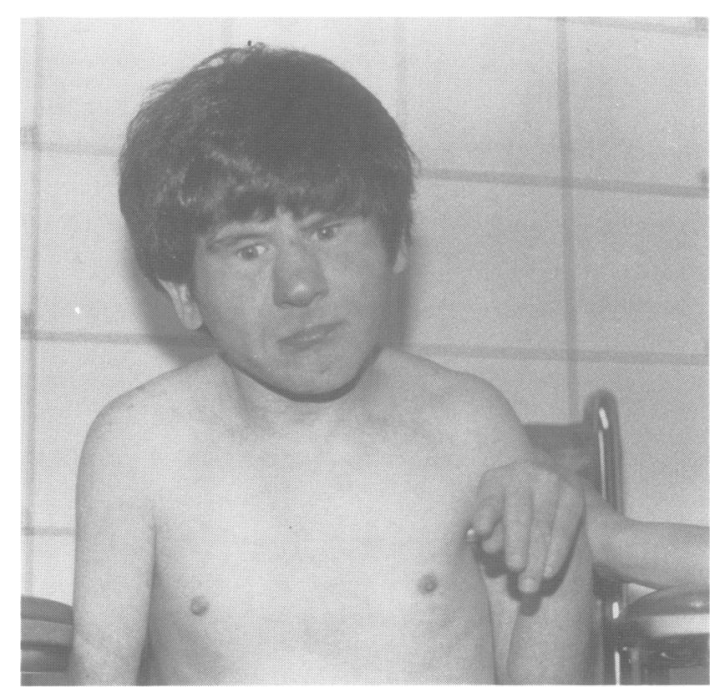

FIGURE The patient aged 27 years. 